\title{
A novel pre-processing and adaptive statistical threshold for sphere detection in MIMO systems
}

\author{
Haiyan Cao ${ }^{1,2}$, Jun $\mathrm{Li}^{3^{*}}$, Xin Fang ${ }^{1}$ and Xiumin Wang ${ }^{3}$
}

\begin{abstract}
In this paper, an efficient sphere detection (SD) scheme for multiple-input multiple-output (MIMO) systems is presented. The proposed SD scheme can substantially reduce computational complexity by introducing a pre-processing scheme and an adaptive threshold. In particular, our proposed pre-processing scheme is based on an iterative successive interference cancelation (SIC). The proposed adaptive threshold is used to judge whether a traversed path is correct or not and obtained by analyzing the distributions of the correct and erroneous estimates which have the minimum error pattern among all the SD searching paths. Simulation results show that our proposed scheme offers a substantial complexity reduction in terms of the number of visited nodes while keeping a significantly small performance degradation compared to conventional approaches.
\end{abstract}

\section{Introduction}

The maximum likelihood (ML) detection is considered as an optimal solution for achieving the minimum error probability of multiple-input multiple-output (MIMO) systems. However, the ML detection, also a well-known non-deterministic polynomial-time (NP)-complete problem, comes at an expense of computational complexity. To overcome this shortcoming, several suboptimal detection algorithms, such as zero forcing (ZF), minimum mean-square error (MMSE), and successive interference cancelation (SIC), have been proposed. Although these algorithms have lower complexity than ML, the achievable performance is far below the performance of the ML receiver.

Sphere detection (SD) algorithm is a tree search strategy that can achieve a similar performance to that of ML and is widely considered to be the most promising approach for MIMO detection. It has polynomial expected complexity for problems with moderate size and can be efficiently used for moderate signal-to-noise ratios (SNRs) $[1,2]$. However, the expected complexity is exponential in the number of antennas and constellation size for fixed

\footnotetext{
${ }^{*}$ Correspondence: roage.li@gmail.com

${ }^{3}$ College of Information Engineering, China Jiliang University, Hangzhou

310018, China

Full list of author information is available at the end of the article
}

SNRs [2], and it still requires a large amount of computations compared to MMSE- and ZF-based algorithms, especially for high numbers of antennas and large constellation sizes. Hence, many efforts have been made to modify the SD algorithm to further reduce the computational complexity such as the Schnorr-Euchner (SE) [3] strategy, channel matrix pre-processing (ordering scheme) [4-6], modification of the lattice representation [7], probabilistic tree pruning strategy [8], and k-best algorithm [9]. All the schemes can improve SD efficiency to some degrees while still maintaining a large amount of computations.

Motivated by all of the above, in this paper, we propose an efficient ordering scheme and adaptive threshold to decrease SD complexity while keeping the system performance satisfactory. An ordering scheme based on SIC which the authors call sorted SIC (SSIC) ordering is proposed. Then, an adaptive threshold which can be seen as the extension of the threshold used in fixed-complexity SD (FSD) [10] is developed. Simulation results show that the proposed scheme combining SSIC ordering with adaptive threshold results in almost minimum complexity (in terms of the number of visited nodes) compared to other existing approaches with neglected performance degradation.

Throughout this paper, the following notations are adopted. Bold uppercase and lowercase letters stand for matrices and vectors, respectively. $(\bullet)^{T}$ and $(\bullet)^{-1}$ describe

\section{望 Springer}

(c) 2013 Cao et al: licensee Springer. This is an Open Access article distributed under the terms of the Creative Commons Attribution License (http://creativecommons.org/licenses/by/2.0), which permits unrestricted use, distribution, and reproduction in any medium, provided the original work is properly cited. 
the transpose and inverse of a matrix, respectively. Furthermore, $\|\bullet\|$ and $|\bullet|$ denote the norm and absolute operator, respectively, and $E[\bullet]$ is the expectation operator. In addition, $(\bullet)_{i}$ means the $i$ th row for a matrix or the $i$ th element of a vector.

\section{System model and sphere detection}

Consider a MIMO system with $N$ transmit and $M$ receive antennas where $M \geq N$. The input-output relationship can be expressed as

$$
\mathbf{y}=\mathbf{H s}+\mathbf{w}
$$

where $\mathbf{s}=\left(s_{1}, s_{2}, \ldots, s_{N}\right) \in \mathcal{C}^{N}$ is an $N$-dimensional transmitted complex vector whose entries $s_{i}, i=$ $1,2, \ldots, N$, are drawn from some quadrature amplitude modulation (QAM) constellation with the real and imaginary parts being integers; $\mathbf{y}=\left(y_{1}, y_{2}, \ldots, y_{M}\right) \in \mathcal{C}^{M}$ is an $M$-dimensional received complex vector; $\mathbf{H} \in \mathcal{C}^{M \times N}$ denotes the $M \times N$ MIMO channel matrix; and $\mathbf{w} \in \mathcal{C}^{N}$ is the additive white Gaussian noise (AWGN) vector. The matrix $\mathbf{H}$ is defined by $\mathbf{H}=\left[h_{i j}\right]$ for $1 \leq i \leq M$ and $1 \leq j \leq N$, where $h_{i j}$, the $i$ th row and $j$ th column entry of $\mathbf{H}$, is assumed to be an independent and identically distributed (i.i.d.) circular symmetric complex Gaussian random variable with 0 mean and 1 variance. The tuples of $N$-dimensional Gaussian noise vector $\mathbf{w}$ are i.i.d. circular symmetric complex Gaussian random variables with 0 mean and $\sigma^{2}$ variance. Herein, the SNR at the receiver is defined as

$$
\mathrm{SNR}=\frac{E\left[\|\mathbf{s}\|^{2}\right]}{E\left[\|\mathbf{w}\|^{2}\right]}=\frac{E\left[\|\mathbf{s}\|^{2}\right]}{N \sigma^{2}} .
$$

For simplicity, the complex-valued model given in (1) is usually transformed into a real-valued model, where the dimension is doubled such as $m=2 M$ and $n=2 N$. As such, (1) can be rewritten as

$$
\left[\begin{array}{c}
\Re(\mathbf{y}) \\
\mathfrak{I}(\mathbf{y})
\end{array}\right]=\left[\begin{array}{cc}
\Re(\mathbf{H}) & -\mathfrak{I}(\mathbf{H}) \\
\mathfrak{I}(\mathbf{H}) & \Re(\mathbf{H})
\end{array}\right]\left[\begin{array}{c}
\Re(\mathbf{s}) \\
\mathfrak{I}(\mathbf{s})
\end{array}\right]+\left[\begin{array}{l}
\Re(\mathbf{w}) \\
\mathfrak{I}(\mathbf{w})
\end{array}\right],
$$

where $\Re(\bullet)$ and $\Im(\bullet)$ denote the real and imaginary parts of complex numbers, respectively. Since all the complex form of (1) can be written as (3), we assume that (1) is a real-valued model without loss of generality in the following. Suppose perfect channel knowledge is available at the receiver, the ML detection problem can be formulated as

$$
\mathbf{s}_{\mathrm{ML}}=\underset{\hat{\mathbf{s}} \in \mathcal{D}}{\arg \min }\|\mathbf{y}-\mathbf{H} \hat{\mathbf{s}}\|^{2},
$$

where $\|\bullet\|$ denotes the Euclidean norm and $\mathcal{D}=\mathcal{A}^{n}$ and $A$ is the signal constellation set, e.g., $\mathcal{A}=\{-3,-1,1,3\}$ for $16 \mathrm{QAM}$. For a general $\mathbf{H}$, this problem is known to be NPhard. Sphere decoding solves (4) by searching only over those points that satisfy a constraint of the form

$$
\|\mathbf{y}-\mathbf{H} \hat{\mathbf{s}}\|^{2} \leq d_{0}^{2} .
$$

In other words, the SD algorithm only searches the lattice points inside the hypersphere centered at the received vector with radius $d_{0}$ instead of searching all lattice points. By applying QR decomposition to $\mathbf{H}$, (5) can be written as

$$
\begin{aligned}
& \left\|\mathbf{y}-\mathbf{Q}\left[\begin{array}{c}
\mathbf{R} \\
\mathbf{0}_{(m-n) \times n}
\end{array}\right] \hat{\mathbf{s}}\right\|^{2} \\
& =\left\|\mathbf{Q}_{1}^{T} \mathbf{y}-\mathbf{R} \hat{\mathbf{s}}\right\|^{2}+\left\|\mathbf{Q}_{2}^{T} \mathbf{y}\right\|^{2} \leq d_{0}^{2},
\end{aligned}
$$

where $\mathbf{R}$ is an $n \times n$ upper triangular matrix, $\mathbf{Q}=\left[\begin{array}{ll}\mathbf{Q}_{\mathbf{1}} & \mathbf{Q}_{\mathbf{2}}\end{array}\right]$ is an $m \times m$ orthogonal matrix, and $\mathbf{Q}_{\mathbf{1}}$ and $\mathbf{Q}_{\mathbf{2}}$ represent the first $n$ and last $m-n$ orthonormal columns of $\mathbf{Q}$, respectively. Suppose radius $d_{0}$ is sufficiently large so that the ML solution lies in the hypersphere, and let $d^{2}=d_{0}^{2}-\left\|\mathbf{Q}_{2}^{\mathrm{T}} \mathbf{y}\right\|^{2}$ and $\mathbf{z}=\mathbf{Q}_{1}^{T} \mathbf{y}=\mathbf{R s}+\mathbf{Q}_{1}^{T} \mathbf{w}$; thus, sphere decoding solves (4) by

$$
\begin{aligned}
\mathbf{s}_{\mathrm{ML}} & =\arg \min _{\hat{\mathbf{s}} \in \mathcal{D}}\|\mathbf{z}-\mathbf{R} \hat{\mathbf{s}}\|^{2} \\
& =\arg \min _{\hat{s}_{j} \in \mathcal{A}}\left(\sum_{i=n}^{1}\left|z_{i}-\sum_{j=i}^{n} R_{i j} \hat{s}_{j}\right|^{2} \leq d^{2}\right),
\end{aligned}
$$

where $R_{i j}$ denotes the $i$ th row and $j$ th column tuple of matrix R. From (7), we can see that $\mathbf{s}_{\mathrm{ML}}$ can be solved recursively by following a similar fashion to that in the SIC technique. Specifically, we use a constrained tree search through multiple levels, starting from $\hat{s}_{n}$ down to $\hat{s}_{1}$, as shown in Figure 1.

\section{Proposed scheme}

In this section, we propose the SSIC ordering scheme to significantly improve the ML performance using the SD algorithm. In addition, we further reduce the SD complexity by developing a new adaptive threshold to judge whether an estimate is correct or not, attempting to output the good estimate as early as possible.

\subsection{Proposed pre-processing}

It has been shown that the SD complexity is highly sensitive to the order of the columns of the channel matrix, which is dependent on both the channel matrix and the received signal. Reordering the channel matrix columns is a simple and efficient pre-processing to reduce the SD complexity while maintaining reliability of the system performance. Some channel matrix reordering approaches, such as V-BLAST ordering [4], balanced sorted QR decomposition (BSQRD) ordering [5,9], and gradientbased (GB) ordering [6], have been proposed to improve the SD efficiency. Good ordering schemes should exploit properties of the channel matrix and the received signal as fully as possible. Among these ordering approaches, ZF is the only simple and efficient reference for ordering. However, the reliability of ZF is highly sensitive to the noise 


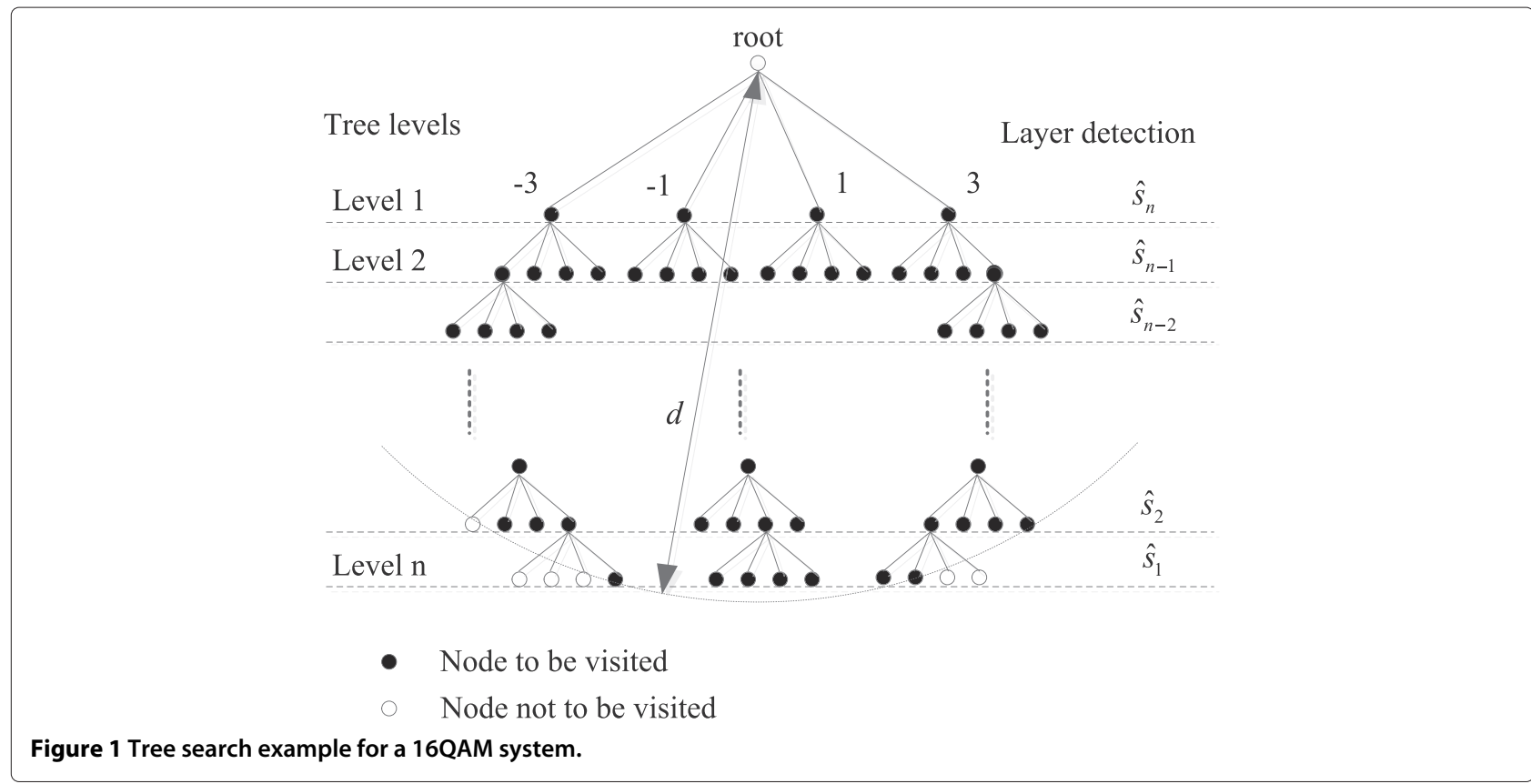

component, which is amplified by the row norm of the pseudo-inverse of the channel matrix as

$$
\mathbf{H}^{\dagger} \mathbf{z}=\mathbf{s}+\mathbf{H}^{\dagger} \mathbf{v}
$$

where $\mathbf{H}^{\dagger}=\left(\mathbf{H}^{T} \mathbf{H}\right)^{-1} \mathbf{H}^{T}$ is the pseudo-inverse of channel matrix $\mathbf{H}$, and $\mathbf{v}=\mathbf{Q}_{1}^{T} \mathbf{w}$ is an i.i.d. noise vector with the same distribution as $\mathbf{w}$. Therefore, the $i$ th tuple of $\mathrm{ZF}$ solutions is given by

$$
\left(\mathbf{H}^{\dagger} \mathbf{z}\right)_{i}=s_{i}+\left(\mathbf{H}^{\dagger}\right)_{i} \mathbf{v}, \quad i=1,2, \ldots, n,
$$

where $\left(\mathbf{H}^{\dagger}\right)_{i}$ denotes the $i$ th row of matrix $\mathbf{H}^{\dagger}$. It is shown that each component of the ZF solution vector is detected out of various noise levels which is modified by $\left(\mathbf{H}^{\dagger}\right)_{i}$. Motivated by the observation, we define the signal reliability:

$$
\operatorname{reliability}\left(s_{i}\right)=\frac{\left\|\left(\mathbf{H}^{\dagger} \mathbf{z}\right)_{i}-s_{i}\right\|}{\left\|\left(\mathbf{H}^{\dagger}\right)_{i}\right\|},
$$

where $s_{i} \in \mathcal{A}$. Based on (10) and due to the similar operation between SD and SIC, the proposed SSIC ordering is described as follows (for $i=0,1, \ldots, n-1$ ):

1. Set $\mathbf{H}_{0}=\mathbf{H}, \mathbf{z}_{0}=\mathbf{z}$, and $\mathcal{N}=\{1,2, \ldots, n\}$.

2. Calculate the matrix $\mathbf{H}_{i}^{\dagger}$, where $\mathbf{H}_{i}^{\dagger}$ is the pseudo-inverse of the $i$ th iteration channel matrix $\mathbf{H}_{i}$.

3. Calculate the ZF solution vector $\mathbf{s}_{i}^{z f}=\left[\left(s_{i}^{z f}\right)_{1}\right.$, $\left.\left(s_{i}^{z f}\right)_{2}, \ldots,\left(s_{i}^{z f}\right)_{n-i}\right]^{T}=\mathbf{H}_{i}^{\dagger} \mathbf{z}_{i}$, where $\mathbf{z}_{i}$ is the $i$ th iteration received signal, and for each element $\left(s_{i}^{z f}\right)_{j}$ of $\mathbf{s}_{i}^{z f}$, find $s_{j}^{(1)} \in \mathcal{A}$ such that $\left\|s_{j}^{(1)}-\left(s_{i}^{z f}\right)_{j}\right\| \leq \| s_{j}^{(\ell)}-$ $\left(s_{i}^{z f}\right)_{j} \|$ holds for any $\ell=2,3, \ldots, L$, where $s_{j}^{(\ell)} \in \mathcal{A}$ and $L$ is the constellation size.

4. For each element $\left(s_{i}^{z f}\right)_{j}$, find out $\hat{k}_{i}=\underset{j=1, \ldots, n-i}{\arg \max }$ $\frac{\left\|s_{j}^{(1)}-\left(s_{i}^{z f}\right)_{j}\right\|}{\left\|\left(\mathbf{H}_{i}^{\dagger}\right)_{j}\right\|}$, and $k_{i}=\mathcal{N}\left(\hat{k}_{i}\right)$, the $\hat{k}_{i}$ th element in set $\mathcal{N}$, is the corresponding column index of channel matrix $\mathbf{H}$, and let $\mathcal{N}=\mathcal{N} \backslash\left\{\hat{k}_{i}\right\}$, where $\backslash$ denotes the set subtraction.

5. Cross the $\hat{k}_{i}$ th column out of $\mathbf{H}_{i}$ to be $\mathbf{H}_{i+1}$, let $\mathbf{z}_{i+1}=\mathbf{z}_{i}-\mathbf{h}_{k_{i}} s_{k_{i}}^{(1)}, i=i+1$, and repeat steps 2 to 5 until $i=n-1$, where $\mathbf{h}_{k_{i}}, i=0,1, \ldots, n-1$ is $k_{i}$ th column of $\mathbf{H}$.

6. The columns of the channel matrix are reordered as $\mathbf{H}=\left[\mathbf{h}_{k_{n-1}}, \mathbf{h}_{k_{n-2}}, \ldots, \mathbf{h}_{k_{1}}, \mathbf{h}_{k_{0}}\right]$.

\subsection{Proposed adaptive threshold}

In the tree searching, each full traversed path metric is calculated by

$$
C=\|\mathbf{z}-\mathbf{R} \hat{\mathbf{s}}\|^{2}=\|\mathbf{R}(\mathbf{s}-\hat{\mathbf{s}})+\mathbf{v}\|^{2} .
$$

When the estimate is correct, i.e., $\hat{\mathbf{s}}=\mathbf{s}, C=\|\mathbf{v}\|^{2}$ is a central chi-square-distributed random variable with $n$ degrees of freedom denoted as $\chi_{n}^{2}$. In contrast, the metric for the case of $\hat{\mathbf{s}} \neq \mathbf{s}$ is the non-central chi-squaredistributed random variable with $n$ degrees of freedom. Given a certain error pattern, if the Hamming distance 
between signal vector $\mathbf{s}$ and estimated vector $\hat{\mathbf{s}}$ is $K$, then $\mathbf{s}$ and $\hat{\mathbf{s}}$ differ at $K$ places denoted as $i_{1}, i_{2}, \ldots, i_{K}$. In this case, the metric $C$ now turns to be $C^{\prime}$ given by

$$
C^{\prime}=\|\mathbf{R}(\mathbf{s}-\hat{\mathbf{s}})+\mathbf{v}\|^{2}=\left\|\sum_{k=1}^{K} \mathbf{r}_{i_{k}}\left(s_{i_{k}}-\hat{s}_{i_{k}}\right)+\mathbf{v}\right\|^{2},
$$

where $\mathbf{r}_{i_{k}}$ is the $i_{k}$ th column of $\mathbf{R}$. Obviously, $\sum_{k=1}^{K} \mathbf{r}_{i_{k}}\left(s_{i_{k}}-\hat{s}_{i_{k}}\right)+\mathbf{v}$ is a Gaussian vector with the mean vector $\sum_{k=1}^{K} \mathbf{r}_{i_{k}}\left(s_{i_{k}}-\hat{s}_{i_{k}}\right)$ and the same covariance matrix as $\mathbf{v}$. Thus, $C^{\prime}$ is the non-central chi-square-distributed random variable with $n$ degrees of freedom denoted as $\chi_{n, \gamma^{2}}^{2}$, where the non-central parameter $\gamma^{2}$ is shown as

$$
\gamma^{2}=\left\|\sum_{k=1}^{K} \mathbf{r}_{i_{k}}\left(s_{i_{k}}-\hat{s}_{i_{k}}\right)\right\|^{2} .
$$

Since the probability density function (PDF) of the metric for correct path is different from that of the metric for erroneous path, the intersection point for PDFs of $\chi_{n}^{2}$ and $\chi_{n, \gamma^{2}}^{2}$ can be set as the optimum threshold whether an estimate is correct or not. Among all the error patterns, the minimum one is that there exists one path which has Hamming distance 1 to the correct path, say, they differ at the site, and the corresponding column of $\mathbf{R}$ exactly has the minimum norm. Therefore, we select the optimum threshold to be the intersection point for PDFs of $\chi_{n}^{2}$ and $\chi_{n, \gamma^{2}}^{2}$, which gives the minimum non-central parameter as

$$
\gamma_{\min }^{2}=\min _{i=1,2, \ldots, n}\left\|\mathbf{r}_{i}\right\|^{2} \times d_{\min }^{2}
$$

where $d_{\min }^{2}=\min _{s, \hat{s} \in A, s \neq \hat{s}}(s-\hat{s})^{2}$ is the minimum squared distance of two constellation points. Based on the noncentral parameter, the threshold can be approximately linearly calculated by [10]

$$
T=\left(\alpha \times \gamma^{2}+n\right) \times \sigma^{2},
$$

where $\alpha$ is a constant coefficient depending on the degrees of freedom, which can be calculated offline by numeric computation since the intersections for PDFs of $\chi_{n}^{2}$ and $\chi_{n, \gamma^{2}}^{2}$ almost lie on a line if the non-central parameters are not very large, as shown in Figure 2 which indicates the PDF for central and non-central $\chi^{2}$ of 8 degrees of freedom. In this way, $\alpha$ can be obtained, for instance, as $0.3465,0.366$, and 0.4082 for the case where the degrees of freedom are 4,8 , and 16 , respectively. The proposed threshold based on minimum error pattern (MEP-T) is applied when the SD searching reaches level $n$ (leaf nodes). Based on our SSIC ordering scheme and proposed threshold, our SD search process can be described as follows:

1. Initialize radius $d \rightarrow \infty$.

2. Perform SSIC precessing algorithm to reorder the columns of channel matrix $\mathbf{H}$.

3. Apply QR decomposition to the reordered channel matrix, i.e., $\mathbf{H}=\mathbf{Q R}$.

4. Calculate threshold $T$ by (15).

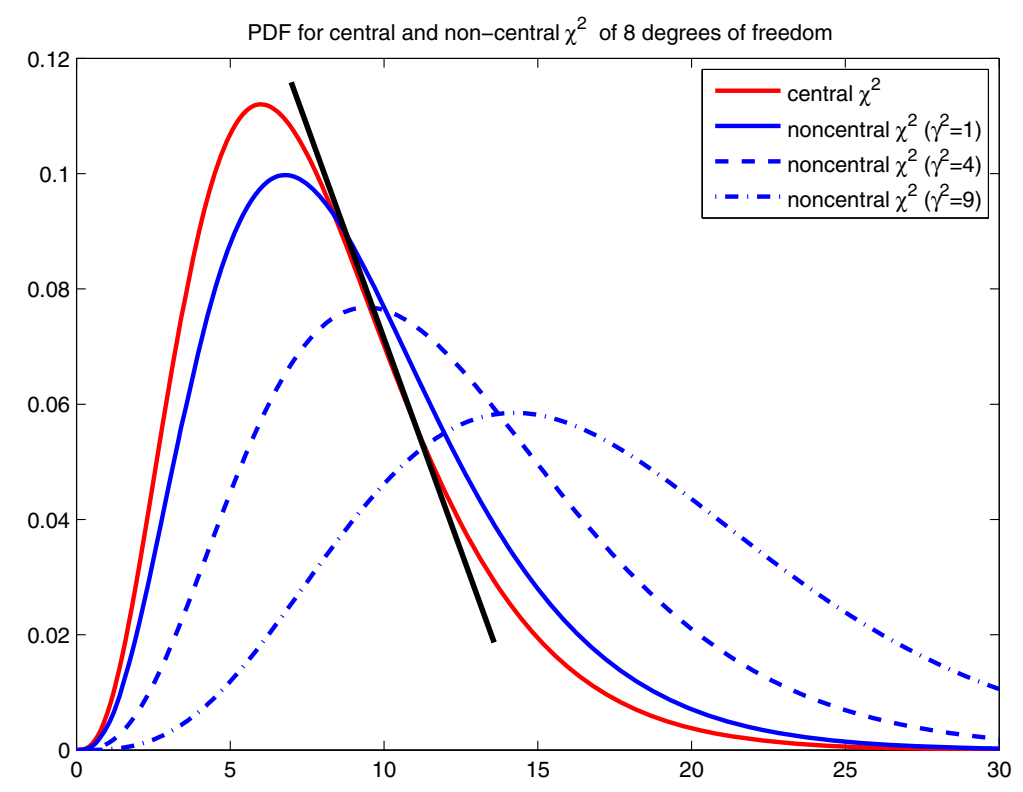

Figure 2 PDF for central and non-central distribution. PDF for central and non-central $\chi^{2}$ of 8 degrees of freedom. 


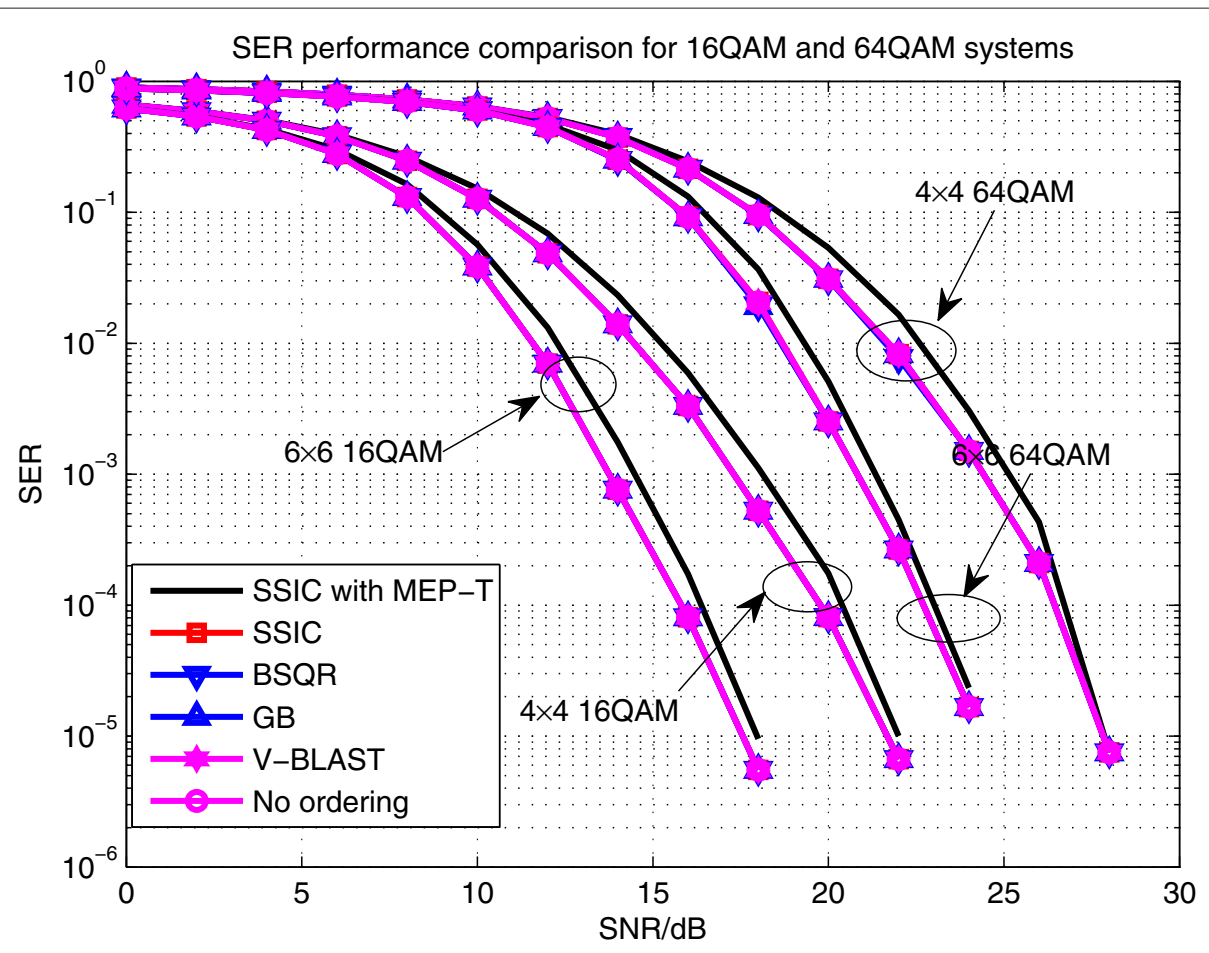

Figure 3 SER performance comparison for 16QAM and 64QAM systems.

5. Normally run a depth-first constrained tree search through $n$ levels.

6. Compute the whole accumulated metric $C$ through $n$ levels by (11), and compare $C$ with threshold $T$.

Terminate the SD decoding process and output the solution if $C \leq T$ holds.

7. Otherwise, compare $C$ with radius $d$. If $C \leq d$, update radius $d$ as $d=C$ and repeat steps from 5 to 7 until a path, which is the first one such that $C \leq T$ holds or has the minimum accumulated metric $C$, is found.

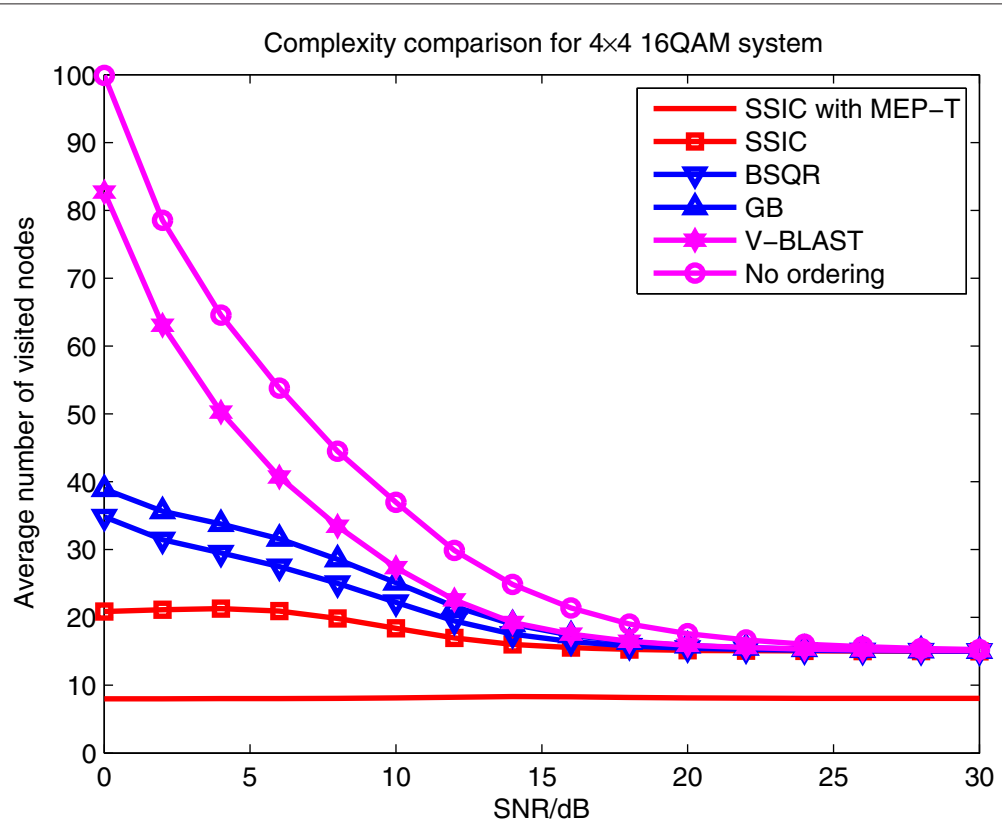

Figure 4 Complexity comparison for a 4 × 4 16QAM system. 


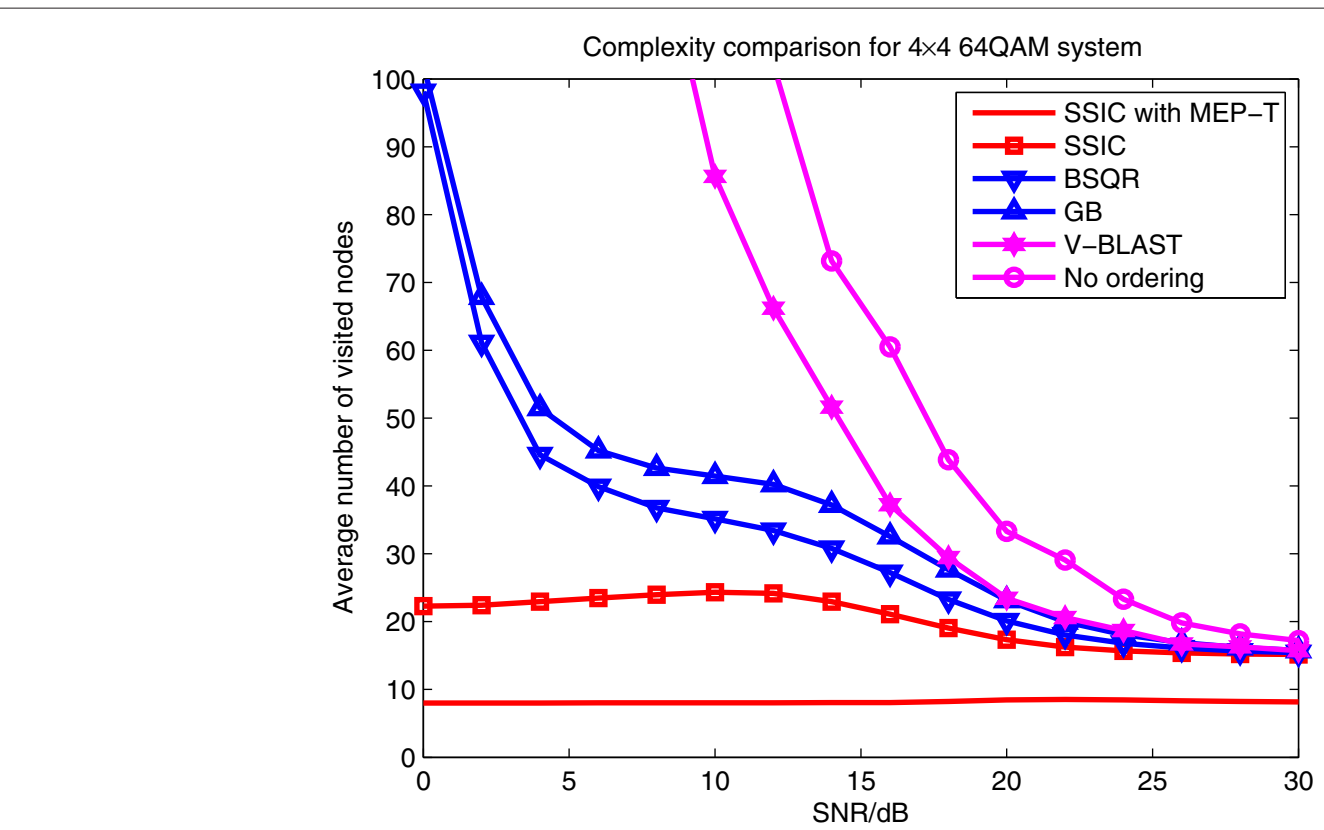

Figure 5 Complexity comparison for a 4 × 4 64QAM system.

\section{Simulation results}

In this section, we display the comparisons of system performance in terms of symbol error rate (SER) and the computational complexity in terms of the average numbers of visited nodes for $4 \times 4$ and $6 \times 6 \mathrm{MIMO}$ systems with 16QAM and 64QAM modulation. As can be observed from Figure 3, SD with our SSIC ordering and MEP-T show very small SER degradation compared to the original SD. However, a considerable complexity reduction can be observed from Figures 4, 5, 6, and 7 . It is clearly shown that our SSIC ordering itself is much more efficient than other conventional approaches such as BSQR, GB, and V-BLAST ordering. Moreover, combining SSIC ordering with MEP-T can further reduce the complexity and offer almost fixed complexity in average. Specifically, for SNR $=5 \mathrm{~dB}$, our proposed

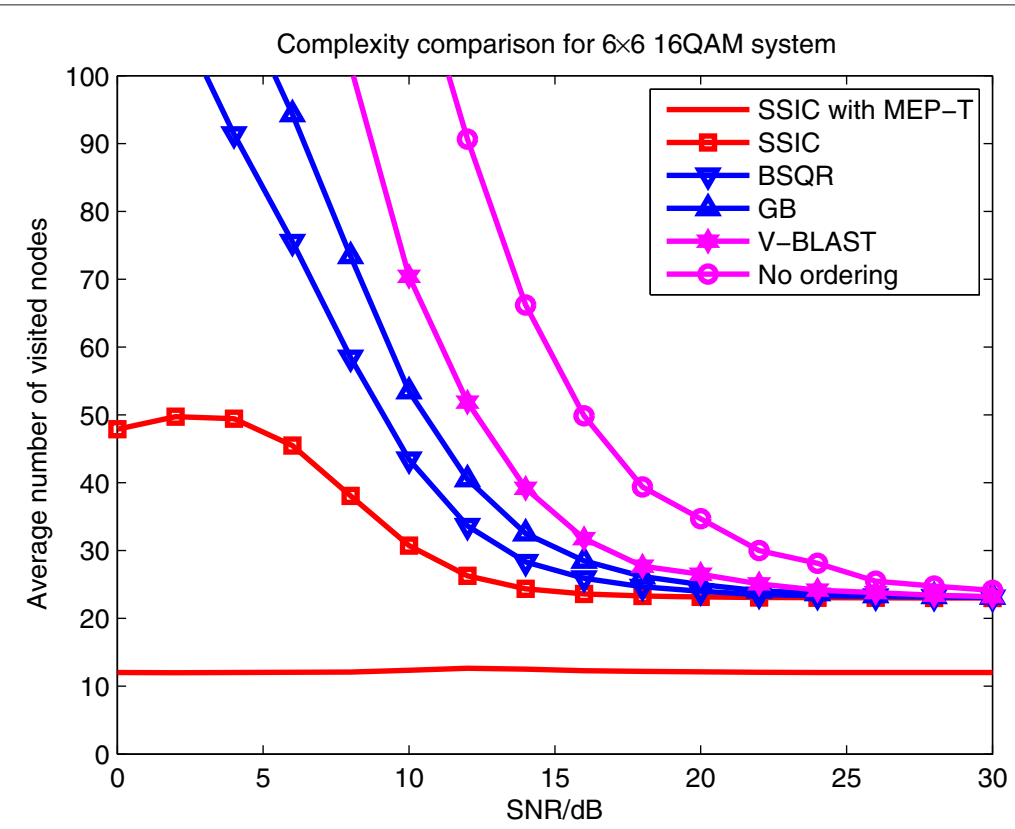

Figure 6 Complexity comparison for a $6 \times 6$ 16QAM system. 


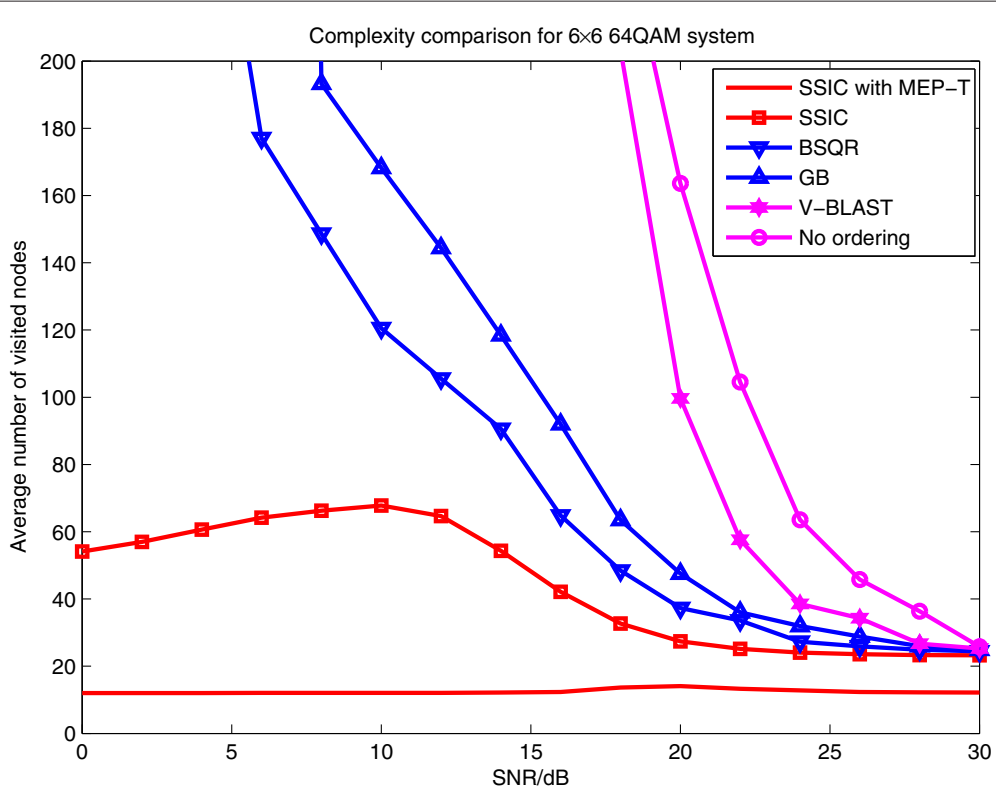

Figure 7 Complexity comparison for a 6 × 6 64QAM system.

scheme yields a little bit more than 8 visited nodes for a $4 \times 4$ system with 16QAM (Figure 4) and 64QAM (Figure 5) and a little more than 12 visited nodes for $6 \times 6$ 16QAM (Figure 6) and 64QAM (Figure 7), whereas other approaches require at least 25 visited nodes or even higher.

Intuitively, the reason why it has such a low complexity in average can be investigated through the behavior of the SSIC ordering and MEP-T threshold. The SSIC ordering leads to the case that the lattice point which has the most possible to be the solution has the higher privilege to be searched. Moreover, the threshold is set properly and can be considered that it constrains a hypersphere area in which all the lattice points (corresponding to the full path in the searching tree) are the 'ML solution.' Since the real ML solution has the minimum metric, it certainly lies in the area. Once any one lattice point in the area has been searched, the searching process terminates. As the SNR increases, the number of lattice points in the area goes down, and the probability of obtaining the real ML solution goes larger and larger. That is why the complexity is low while having a negligible performance loss at the highSNR region under our scheme. At the low-SNR region, the area contains more lattice points, which leads to an incorrect estimate with a larger probability. However, the errors given in this way do not affect the performance significantly because even the real ML solution yielded by the exhaustive search at the low-SNR region also gives the wrong estimate. Therefore, in our SSIC with MEP-T scheme, the correct and incorrect estimates are well distinguished at the high-SNR region, and the complexity reduction is preferred at the low-SNR region instead of improving a little performance while increasing enormous complexity.

\section{Conclusions}

In this paper, we propose an efficient SD algorithm by jointly combining the SSIC ordering scheme and the adaptive threshold MEP-T. In particular, the SSIC ordering scheme is based on the iterative SIC and MEP-T is obtained by analyzing the PDFs of the correct and erroneous estimates, which have the minimum error pattern among all the SD searching paths. Simulation results show that our SSIC ordering scheme is more efficient than BSQR, GB, and V-BLAST by reducing the computational complexity in terms of the number of visited nodes. More importantly, combining SSIC ordering with MEP$\mathrm{T}$ can further reduce the complexity and offer almost a fixed average complexity at an expense of an unnoticeably small performance degradation compared to conventional methods.

\section{Competing interests}

The authors declare that they have no competing interests.

\section{Acknowledgements}

The authors would like to thank the Associate Editor and the reviewers for their comments and suggestions which help improve the quality and clarity of this paper. This work was supported by Zhejiang Natural Science Foundation (Y1090935) and National Natural Science Foundation of China (61379027).

\section{Author details}

${ }^{1}$ College of Communication Engineering, Hangzhou Dianzi University, Hangzhou 310018, China. ${ }^{2}$ School of Information Engineering, Hangzhou Dianzi University, Hangzhou 310018, China. ${ }^{3}$ College of Information Engineering, China Jiliang University, Hangzhou 310018, China. 
Received: 10 May 2012 Accepted: 12 November 2013

Published: 5 December 2013

\section{References}

1. J Jalden, B Ottersten, An exponential lower bound on the expected complexity of sphere decoding, in Proceedings of the IEEE International Conference on Acoustics, Speech, and Signal Processing (IEEE, Montreal, 2004), pp. 393-396

2. J Jalden, B Ottersten, On the complexity of sphere decoding in digital communications. IEEE Trans. Sig. Proc. 53(4), 1474-1484 (2005)

3. CP Schnorr, M Euchner, Lattice basis reduction: improved practical algorithms and solving subset sum problems. Math. Program. 66(2), 181-191 (1994)

4. PW Wolniansky, GJ Foschini, GD Golden, RA Valenzuela, V-BLAST: an architecture for realizing very high data-rates over the rich-scattering wireless channel, in Proceedings of the URSI International Symposium on Signals, Systems, and Electronics (IEEE, Piscataway, 1998), pp. 295-300

5. Y Dai, Z Yan, Memory-constrained tree search detection and new ordering schemes. IEEE J. Sel. Topic Sig. Process. 3(6), 1026-1037 (2009)

6. R Trujillo, VM Garcia, A Vidal, S Roger, A Gonzalez, Gradient-based ordering for MIMO decoding, in Proceedings of the IEEE International Symposium on Signal Processing and Information Technology (ISSPIT) (IEEE, Ajman, 2009), pp. 5-8

7. L Azzam, E Ayanoglu, Reduced complexity sphere decoding via a reordered lattice representation. IEEE Trans. Commun. 57(9), 2564-2569 (2009)

8. S Lei, C Xiong, X Zhang, D Yang, Adaptive control of surviving branches for fixed-complexity sphere decoder, in Proceedings of the IEEE 71st Vehicular Technology Conference (IEEE, Taipei, 2010), pp. 1-5

9. $\mathrm{X} W u, Y$ Dai, Z Yan, Y Wang, Improving the reliability of the k-best algorithm for MIMO detection with ordering, in Proceedings of the Wireless and Optical Communications Conference (WOCC) (IEEE, Shanghai, 2010), pp. 1-5

10. $X W u, J$ Thompson, Accelerated sphere decoding for multiple-input multiple-output systems using an adaptive statistical threshold. IET Signal Process. 3(6), 433-444 (2009)

doi:10.1186/1687-1499-2013-275

Cite this article as: Cao et al:: A novel pre-processing and adaptive statistical threshold for sphere detection in MIMO systems. EURASIP Journal on Wireless Communications and Networking 2013 2013:275.

\section{Submit your manuscript to a SpringerOpen ${ }^{\mathcal{O}}$ journal and benefit from:}

- Convenient online submission

Rigorous peer review

- Immediate publication on acceptance

- Open access: articles freely available online

- High visibility within the field

- Retaining the copyright to your article

Submit your next manuscript at $\gg$ springeropen.com 